\title{
Synthesis and Characterization of Monodisperse Magnetic Nanoparticles by a Scanning Susceptometer
}

Jefferson Araujo $^{1}$, Frederico Gutierrez ${ }^{1}$, Elder Yokoyama ${ }^{2}$, Geronimo Perez $^{3}$ and Guillermo Solórzano ${ }^{4}$

${ }^{1}$ Pontifical Catholic University of Rio de Janeiro, Rio de Janeiro, Brazil, ${ }^{2}$ Institute of Geosciences, University of Brasilia, Brasilia, Distrito Federal, Brazil, ${ }^{3}$ Materials Metrology Division, National Institute of Metrology, Standardization and Industrial Quality (Inmetro), Rio de Janeiro, Rio de Janeiro, Brazil, ${ }^{4}$ Department of Chemical and Materials Engineering, Pontifical Catholic University of Rio de Janeiro, Rio de Janeiro, Brazil

In this article, monodisperse magnetic nanoparticles (MNPs) of magnetite synthesized by co-precipitation were characterized by a magnetic method especially appropriate for low mass samples in the order of tens of micrograms. This method is based on scanning magnetic microscopy (SMM) and has been an important research tool for obtaining the magnetic properties of different materials and for their applications in areas such as geology, biomedicine, science and technology [1-3]. In this study, it was used a susceptometer composed by a reading system based on a gradiometric configuration using two Hall effect sensors made of GaIn with a magnetic detection diameter of $300 \mu \mathrm{m}$. This equipment can generate magnetic maps under an applied magnetic field of up to $500 \mathrm{mT}$. The magnetic moment sensitivity reached a value of the order of $10^{-12} \mathrm{Am}^{2}$. As an application example, an average diameter of monodisperse magnetic nanoparticles of magnetite, synthesized by co-precipitation, was estimated. The results were compared with those obtained by other techniques, such as transmission electron microscopy (TEM) and dynamic light scattering (DLS). The reagents ferric chloride hexahydrate $\left(\mathrm{FeCl}_{3} .6 \mathrm{H}_{2} \mathrm{O}\right)$, ferrous chloride tetrahydrate $\left(\mathrm{FeCl}_{2} .4 \mathrm{H}_{2} \mathrm{O}\right)$, ammonium hydroxide $\left(\mathrm{NH}_{4} \mathrm{OH}, 30 \%\right)$, and hydrochloride acid $(\mathrm{HCl}, 38 \%)$ were used in the synthesis process, which can be summarized as follow: (1) Iron salts preparation, which consisted of preparing two solutions, one by dissolving $6.8 \mathrm{~g}$ of $\mathrm{FeCl}_{3} .6 \mathrm{H}_{2} \mathrm{O}$ in $25 \mathrm{~mL}$ of distilled water, and another by dissolving $3.95 \mathrm{~g}$ of $\mathrm{FeCl}_{2} .4 \mathrm{H}_{2} \mathrm{O}$ in $10 \mathrm{~mL}$ of aqueous $\mathrm{HCl}$ solution $(5.49 \mathrm{M})$. (2) The iron solutions were mixed together in a proportion of $24 \mathrm{~mL}$ of iron (III) to $6 \mathrm{~mL}$ of iron (II), and the obtained mixture was added to an aqueous $\mathrm{NH}_{4} \mathrm{OH}$ solution $(1.30 \mathrm{M})$, heated to $80^{\circ} \mathrm{C}$ for 10 minutes, with vigorous stirring. The iron oxide precipitation was instantly observed, as the solution turned black [4]. After making the nanoparticles, they were characterized using the SMM technique in the susceptometer. This technique is based on the measurement of the dispersed induced magnetic field generated by the samples. In this way, magnetic maps can be obtained for each external magnetic field applied by the equipment, making it possible to obtain the magnetization curve necessary to magnetically characterize the samples (FIG. 1.a and 1.b). In the case of nanoparticles, the magnetization curve shows a superparamagnetic behavior with no remanence and coercivity field (FIG. 1.b). In addition to characterizing the behavior of MNPs (superparamagnetic behavior) at room temperature, the average diameter (around $12 \mathrm{~nm}$ ) was estimated based on the magnetization curve [3]. The average diameter of MNPs is usually obtained by using techniques such as TEM (FIG. 2.a and 2.b), which, in our study, obtained an average diameter of $14 \mathrm{~nm}$ (FIG. 2.c). For the TEM measurements, we used a Tecnai G2 Spirit TWIN FEI micro-scope, USA, operating at $120 \mathrm{kV}$ with a LaB6 (lanthanum hexaboride) filament. The particle size distribution of magnetic nanoparticles was also estimated by DLS. We performed DLS using a Nano SZ-100 HORIBAJapan Scientific nanoparticle analyzer, with an incident beam at a wavelength of $532 \mathrm{~nm}$. The particle has a diameter of $207 \mathrm{~nm}$ (FIG. 2.d). As expected, the diameter of nanoparticles obtained by DLS was larger 
than those obtained from other techniques, because the DLS technique measures the hydrodynamic diameter of suspended particles and aggregates [5].

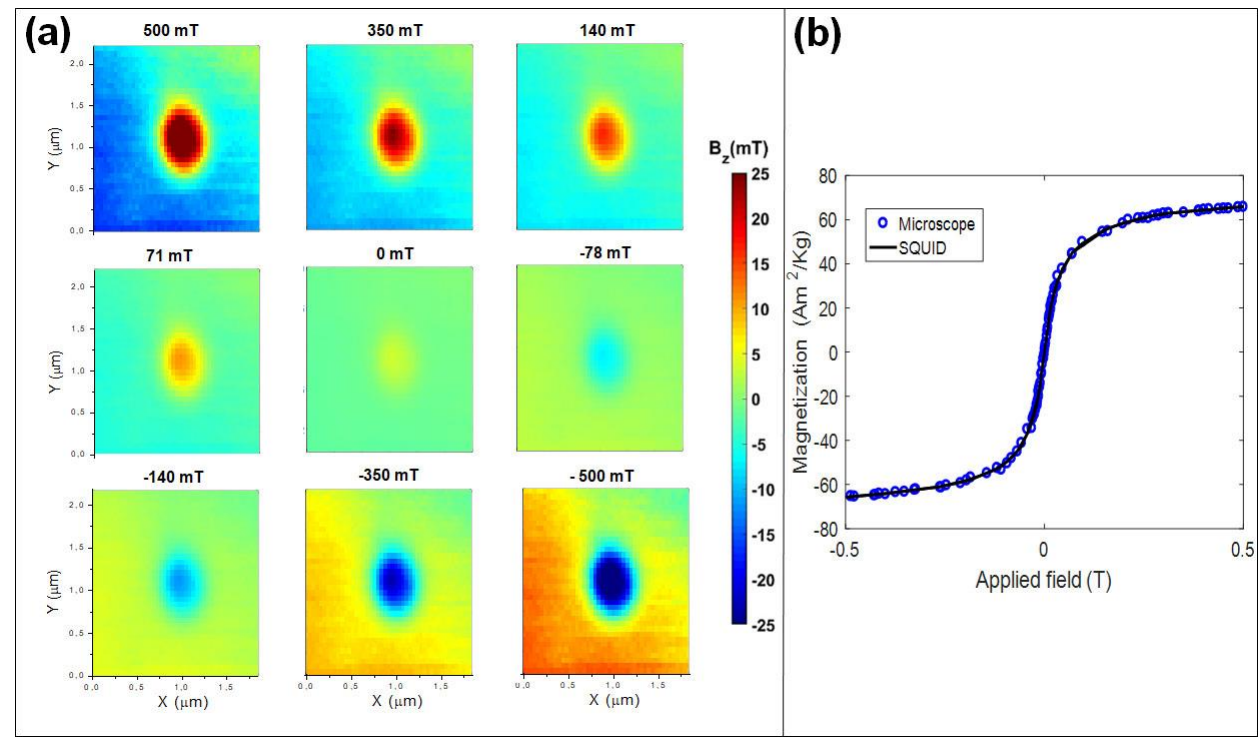

Figure 1. (a) Graphs representing the $1.8 \mathrm{~mm} \times 2.2 \mathrm{~mm}$ mappings of the region surrounding the cylindrical cavity containing a few tens of micrograms of magnetic nanoparticles when applied $500 \mathrm{mT}$ to $-500 \mathrm{mT}$. (b) Magnetization curves of the prepared MNPs at room temperature.

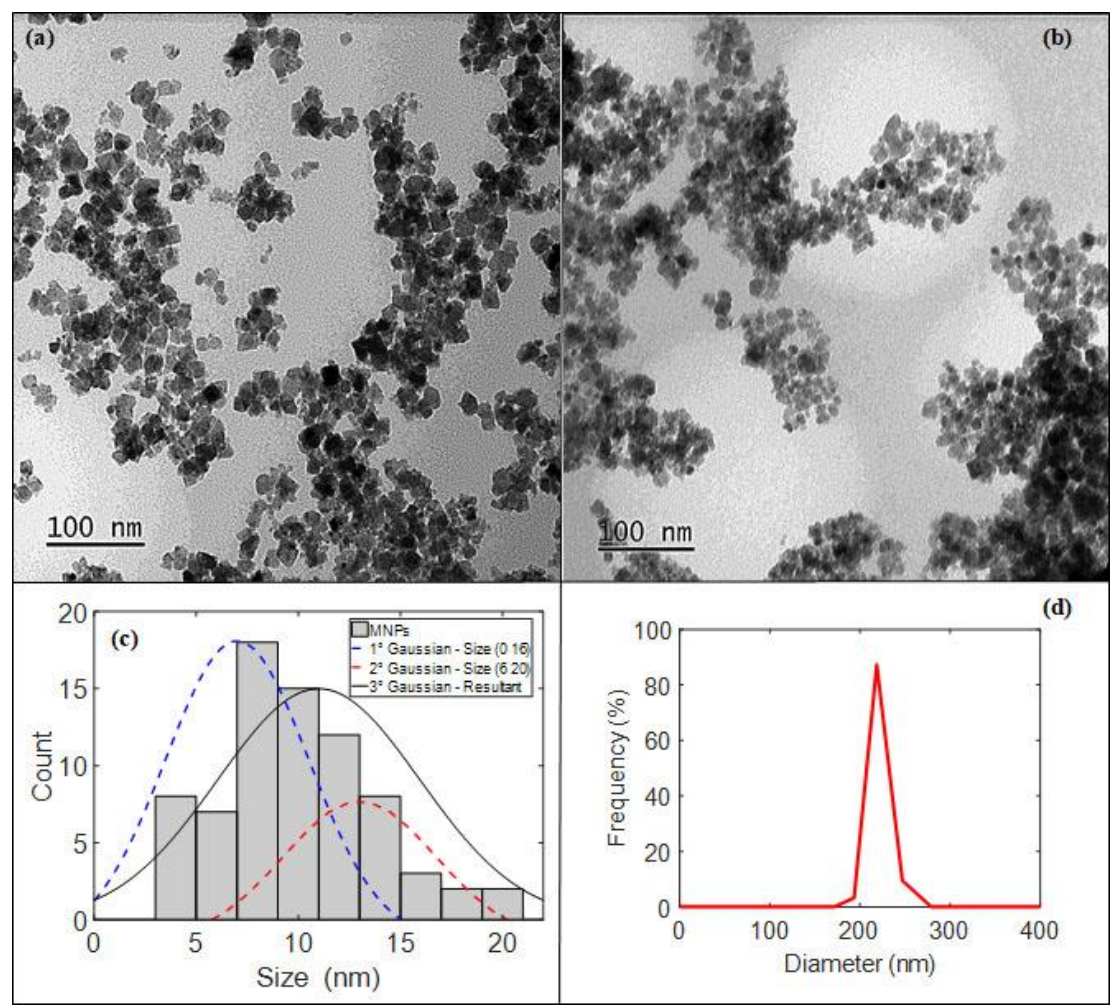

Figure 2. (a) and (b) Bright field TEM images of monodisperse magnetic nanoparticles (MNPs) produced by co-precipitation. (c) Average diameter measured from TEM images. (d) Curve obtained using the DLS technique. 


\section{References}

1. H. Wang, et al., Science 355 (2017) 623-627.

2. M. Wit and G. Welker, J. Magn. Magn. Mater. 98 (2019) 67-70.

3. J. F. D. F. Araujo et al., J. Magn. Magn. Mater. 499 (2020) 166300 - 9.

4. S. Arsalani et al., ACS Sustainable Chem. Eng. 6 (2018) 1-11.

5. Acknowledgments: CNPq (Brazil) and Capes (Brazil) for financial support. 